\title{
Android based Chat-Bot
}

\author{
Shabina Sayed \\ Assistant Professor, \\ Department of Information \\ Technology, \\ MHSSCOE \\ Mumbai
}

\author{
Rushabh Jain \\ Student B.E, Department of \\ Information \\ Technology, \\ MHSSCOE \\ Mumbai
}

\author{
Burhanuddin Lokhandwala \\ Student B.E, Department of \\ Information \\ Technology, \\ MHSSCOE \\ Mumbai
}

\author{
Fakhruddin Barodawala \\ Student B.E, Department of Information \\ Technology, \\ MHSSCOE \\ Mumbai
}

\author{
Murtuza Rajkotwala \\ Student B.E, Department of Information \\ Technology, \\ MHSSCOE \\ Mumbai
}

\begin{abstract}
Today the users face a lot of problem regarding booking of the hotels in any android application because in most of the cases the user gets result more than what he expected or he gets results which are not according to his convenience. This paper focuses on automating the process of communication by use of chat-bot and it also focuses on providing customized results to the user which makes the process of hotel booking convenient and user friendly for him. An extensive research done on existing systems gave us an insight into their shortcomings which this system attempts to overcome by creating a chat-bot using Artificial Intelligence Markup Language and using various algorithms such as Keyword Matching , String Similarity, Spell Checker and Natural language parser. The implementation of this system has resulted in better resource utilization and increased responsiveness of user behavior. This system has been implemented to integrate with any Hotel Management Android Application to ease the process of hotel booking.
\end{abstract}

\section{Keywords}

Chat-bot, Android, Hotel Reservation, Artificial, Intelligence Markup Language

\section{INTRODUCTION}

Android based AI bot can interact with the user after understanding the requirement of user. So instead of a human on one side replying to the queries of another human on the other side the AI bot will act as a human and reply to the queries of the user. AI Bot uses what are called neural networks, vast networks of machines that approximate the web of neurons in the human brain. It is a software application that runs automated tasks over the Internet. Traditionally chatbot performs tasks that are simple and structurally repetitive, at a much higher rate that is possible for a human alone. Bot is a type of interactive agent, a computer program designed to simulate an intelligent conversation with one or more human users via speech recognition and chat interface. The system provides precise output to its users even if minor spelling mistake is there. Moreover the parsing of sentences avoids sending to the system words that do not form a pattern. The project benefits the individuals who regularly travel.

\section{IMPLEMENTATION}

\subsection{Need For The System:}

The communication of potential users is performed manually and it is a very time consuming procedure. The one to one communication between user and the chat-bot is highly valued. However with thousands of applications each year, managing one-to-one conversations are not viable in most cases. It would be time consuming for administrator of an android application to find suitable answers and notify each user. It's useful to reduce this time consuming process. The project aims to reduce the burden on the individual managing the conversation, and potentially other users, by developing a convincing chat-bot. A chat interface is provided for both users and a bot.

\subsection{Existing system:}

- The first chat-bot developed was ELIZA. It was developed by Joseph Weizenbaum using a keyword matching technique. The idea was to read the input from the user and search for specific patterns, if a pattern was found then the answer is retrieved. If a keyword is not found then ELIZA tries, according to defined rules, to get more information from the user to keep the communication with the user alive.

- $\quad$ ALICE uses pattern matching and saves the information in Artificial Intelligence Mark-up Language (AIML) files. Like ELIZA it is a chat-bot that is used mainly for the purpose of chatting with the user. It is mainly used for casual communication with user.

- Natasha is a live assistant on hike android application and replies to your text, in a pre-defined intelligent way. You can spend time with her if you feel bored, she will surely make you feel better. She is a really good assistant who can provide you with quite a lot of useful information

\subsection{Problems in the existing system}

- $\quad$ ELIZA doesn't understand what it is saying. It only produces results according to rules that are predefined.

- $\quad$ ALICE does not have the ability to learn and can only come with data that exist in its database. 
- Natasha is used only for the purpose of chatting with the users and providing information which is available over internet. This system is not used for purpose of reservation of hotels.

In normal hotel booking applications users face difficulties in transitions between transactions. You have to go through multiple screens to process the transaction.

\section{PROPOSED SYSTEM}

This system communicates with the users using a chatting application which provides intelligent answers and guidance to get information required for bookings of hotel rooms. The system provides precise output to its user's even avoiding minor spelling mistake. Moreover the parsing avoids sending the system, words that do not form a patterns. Regular travelers can make most out of the system.

\subsection{GPS Tracking}

GPS tracking is a process that uses the Global Positioning System to determine and track the precise location of the user's device. The application starts with the tracking of location of the logged in user and the location of user is stored in the database. This location is useful whenever the user books a hotel room in same city or in case the user does not mention the location in which he wants the hotel room.

\subsection{AI Calculation}

AI calculation is done by the chat-bot which is scripted in AIML. It starts with providing of input by the user through the chat interface. Now the chat-bot checks whether input provided by the user is inappropriate, insufficient, complete, or conversational. If the input is inappropriate the user is notified that he has entered a wrong input. If the input is insufficient the user is notified to enter the missing parameters. If the input is conversational the chat-bot undergoes casual chat with the user. If the input is complete the user is provided a precise output.

\subsection{Hotel Reservation}

Hotel Reservation starts with customized recommendations to the user. Then the user selects one of the hotel from the above recommendation. Now the user is provided a link for payment and by accessing the link the user is redirected to payment gateway page. Then the user pays for the hotel which he has selected. Now the user is notified his booking details through the chat interface.

\section{IMPLEMENTATION}

The developed android application is used for booking of hotels in a chat interface.

Technologies that have been used include PHP, AIML, Java, Android and SQLite for implementation. The tools used are Android Studio, Program AB and Postman.

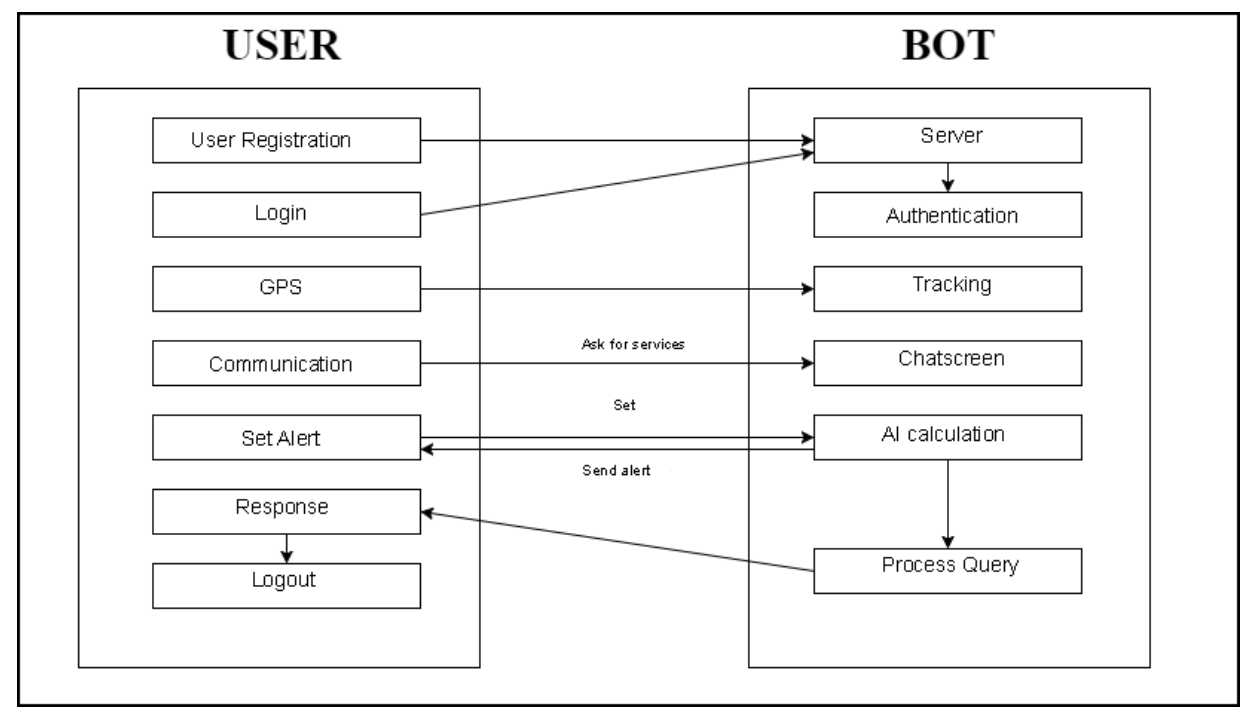

Fig 1: Block Diagram of Android based Chat bot

Figure 1 shows the working of the android based AI bot for hotel booking. Process starts with the authentication of the user. After user is authenticated the location of the user is obtained using the GPS tracking system. Then user gets access to the chat-screen through which he provides his requirement. Based on the requirement the bot fetches the keywords and if it matches with the keywords required for hotel booking, the user is provided with customized recommendation of hotel using hotel database within his budget. If the user agrees with the recommendation then a payment link is provided through which he makes the payment. 


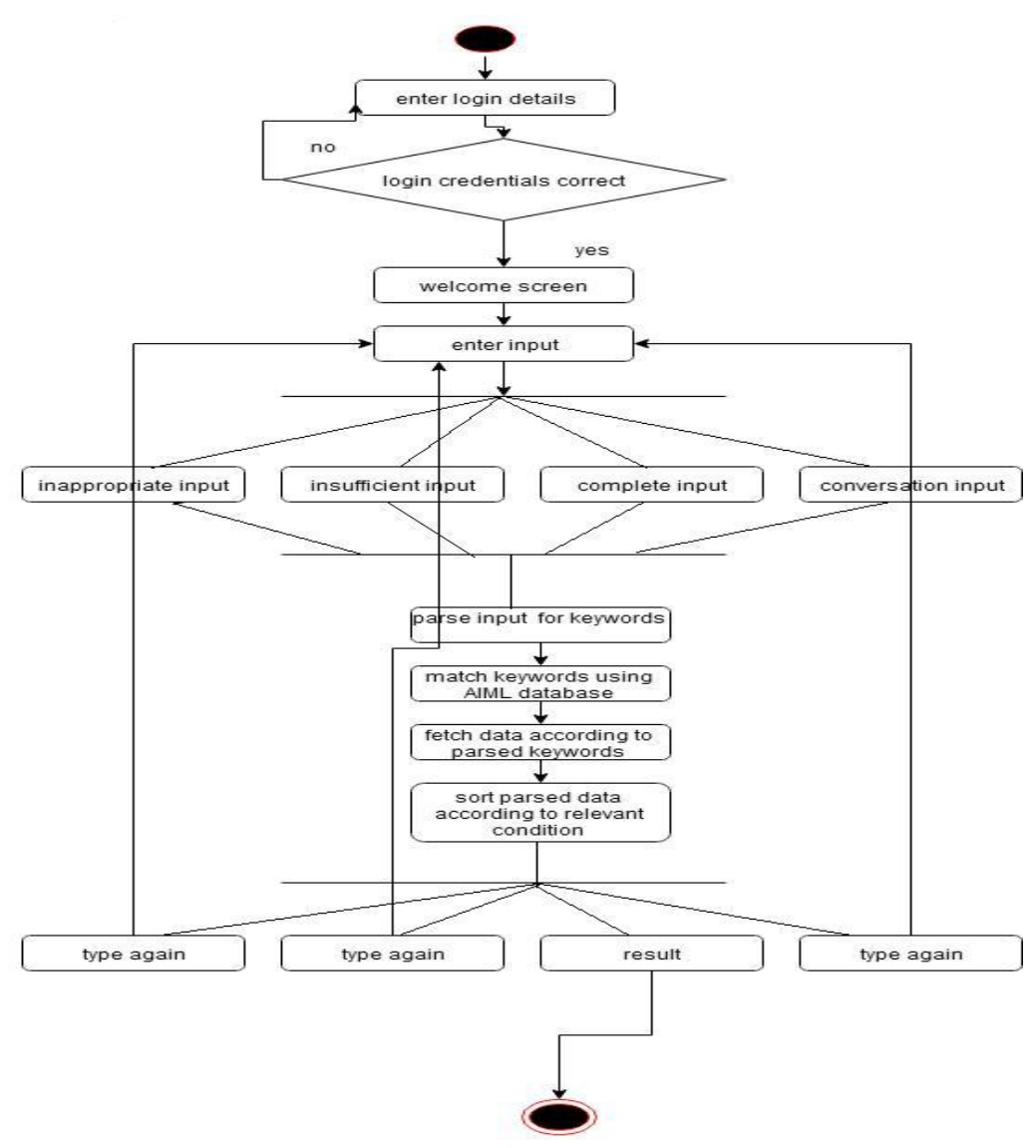

Fig 2: Activity Diagram of Android based Chat-bot

Figure 2 shows the Activity Diagram of the system. When the user enters the input the bot differentiates whether it is inappropriate, insufficient, conversational, or complete input. .
If the input is inappropriate the user is notified that he has entered a wrong input. If the input is insufficient the user is notified to enter the missing parameters. If the input is

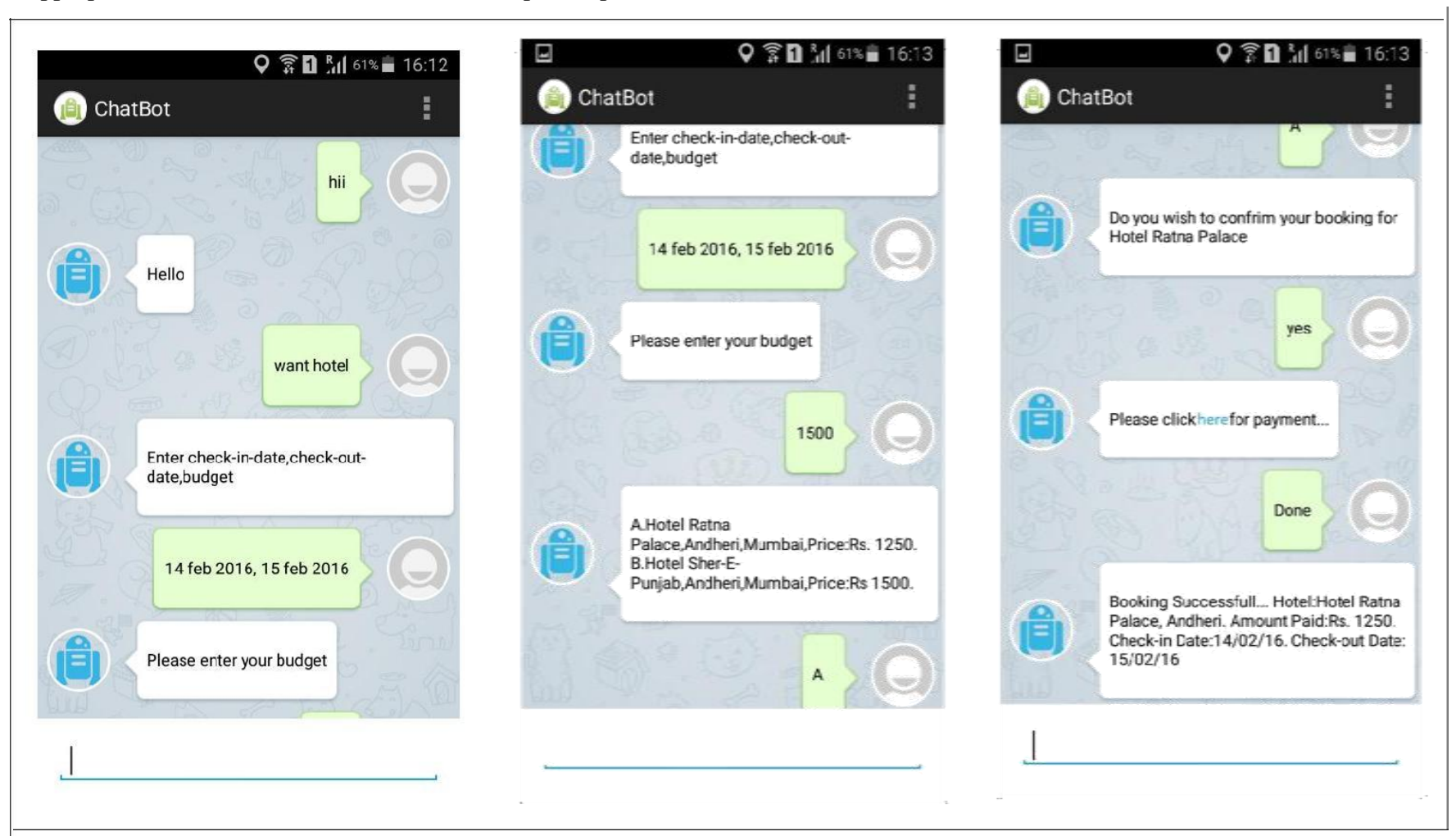

Fig 3: Chat Screen of Android based Chat bot 
conversational the chat-bot undergoes casual chat with the user. If the input is complete the bot parses input for keywords then it matches the keywords based on pattern matching algorithm. Now, the matched keywords are sorted and sent to the hotel database from where the desired response will be generated.

Figure 3 show the user interface of Android based AI bot. The figure shows the communication between the user and the chat-bot which reflects the whole hotel booking procedure using the hotel booking application.

\section{RESULT ANALYSIS}

The benefit of using this system is that it provides us with the following important benefits:
1. This system is time efficient as whole booking procedure is done in a single chat interface.

This system provides the customized output by reducing the confusion of the user related to multiple options

2. This system provides real-time support to the user.

3. This system provides multidimensional evaluation which is considering all the possible aspects related to a particular subject and then coming to a final decision.

4. This system has adaptive and flexible structure related to the different types of input that the user provides.

Table 1. Comparison of Systems

\begin{tabular}{|c|c|c|c|c|}
\hline System & Alice & Eliza & Natasha & $\begin{array}{l}\text { Android based Chat- } \\
\text { bot }\end{array}$ \\
\hline $\begin{array}{l}\text { Conversational } \\
\text { Abilities }\end{array}$ & Yes & Yes & Yes & Yes \\
\hline Real Time Support & No & No & No & Yes \\
\hline Database Support & No & No & No & Yes \\
\hline GPS Tracking & No & No & No & Yes \\
\hline $\begin{array}{l}\text { Multidimensional } \\
\text { Evaluation }\end{array}$ & No & No & No & Yes \\
\hline Human Visual Look & Yes & Yes & Yes & Yes \\
\hline Personalization & No & No & No & Yes \\
\hline Domain Specific & No & No & Yes & Yes \\
\hline Built In knowledge base & Yes & Yes & Yes & Yes \\
\hline Environment & Static & Static & Static & Dynamic \\
\hline
\end{tabular}

12

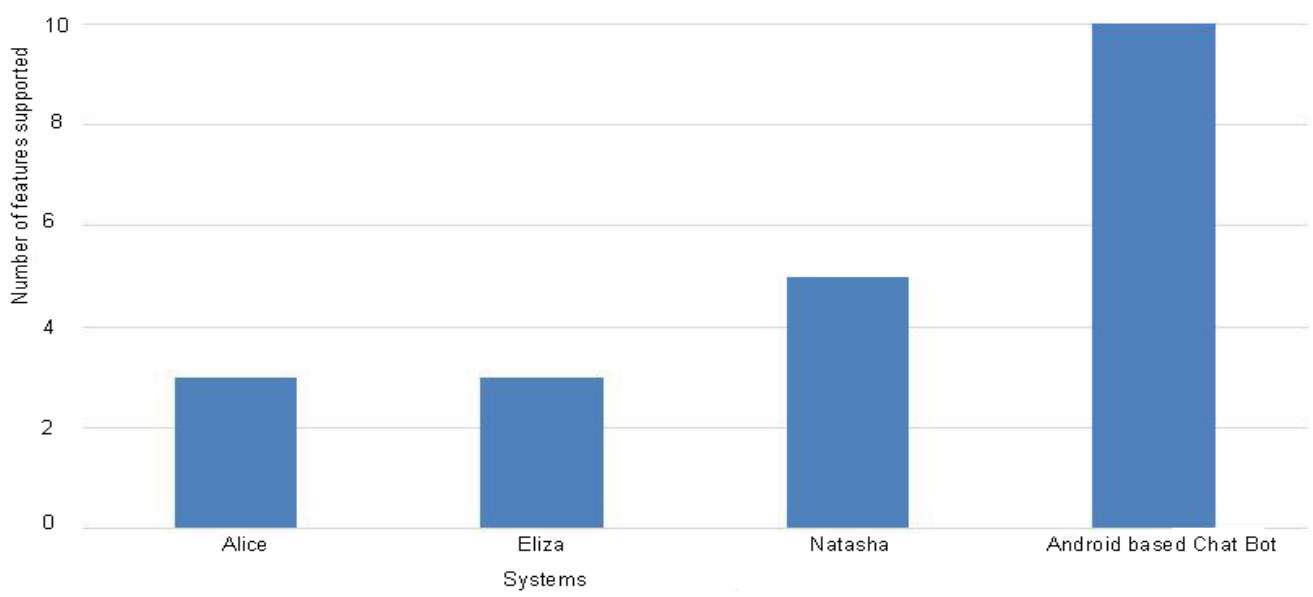

Fig 4. Comparison of Systems 
Table 1 shows the comparison of the existing and the proposed system. Figure 4 shows the graphical representation of table 1.

\section{CONCLUSION}

A chat-bot is one of the easiest way to fetch information from a system without having to think for proper keywords to look up in a search engine or browse several web pages to collect This chat-bot has built-in knowledge-base, conversational abilities, real-time support, and human visual look. It can act as a great virtual assistant for hotel booking and can perform multi-dimensional evaluation. 'Android based smart chat-bot' is a very promising system and aims to enhance the user and customer experience.

\section{REFERENCES}

[1] Walker, Smith, Adrian Charles, "Privatized infrastructure the build operate transfer approach", Thomas Telford. p. 258.(1995)

[2] Bran, "Chabot's in customer communication", Springer, Berlin (2003).

[3] T. Murrari, "Authoring Intelligent Tutoring Systems: An Analysis of the State of the Art", International Journal of Artificial Intelligence in Education, 10, 98-129, (1998). information users can easily type their query in natural language and retrieve information. This project looks into how the chat-bot can be tailored to be used as a domain specific chat-bot, and how can this application make the procedure of hotel booking user-friendly for the android users. Existing chat-bots have various missing aspects that is covered by this system, making it superior.

[4] R.S. Russell, "Language Use, Personality and True Conversational Interfaces." Project Report, AI and CS, University of Edinburgh, Edinburgh, (2002)

[5] Alan Turing , "Computing Machinery and Intelligence," Mind 59: 433-60, doi:10.1093/mind/lix.236.433, (1950)

[6] Weizenbaum, Joseph ,"Computer power and human reason: from judgment to calculation," W. H. Freeman and Company, ISBN 0-7167-0463-3, (1976)

[7] McCorduck, Pamela ," Machines Who Think (2nd ed.)," Natick, MA: A. K. Peters, Ltd., ISBN 1-56881-205-1, (2004)

[8] A. Gracy, T. Butler, "Beyond Knowledge Management: Introducing Learning Management Systems", Idea Group Publishing, (2006) 